\title{
Spectral-Homotopy Perturbation Method for Solving Governing MHD Jeffery-Hamel Problem
}

\author{
Ahmed A. Khidir \\ Faculty of Technology of Mathematical Sciences and Statistics, Al-Neelain University, Algamhoria Street, \\ P.O. Box 12702, Khartoum, Sudan \\ Correspondence should be addressed to Ahmed A. Khidir; ahmed.khidir@yahoo.com
}

Received 20 February 2014; Revised 3 July 2014; Accepted 7 July 2014; Published 14 July 2014

Academic Editor: Xavier Ferrieres

Copyright (C) 2014 Ahmed A. Khidir. This is an open access article distributed under the Creative Commons Attribution License, which permits unrestricted use, distribution, and reproduction in any medium, provided the original work is properly cited.

We present a new modification of the homotopy perturbation method (HPM) for solving nonlinear boundary value problems. The technique is based on the standard homotopy perturbation method and blending of the Chebyshev pseudospectral methods. The implementation of the new approach is demonstrated by solving the MHD Jeffery-Hamel flow and the effect of MHD on the flow has been discussed. Comparisons are made between the proposed technique, the previous studies, the standard homotopy perturbation method, and the numerical solutions to demonstrate the applicability, validity, and high accuracy of the presented approach. The results demonstrate that the new modification is more efficient and converges faster than the standard homotopy perturbation method at small orders. The MATLAB software has been used to solve all the equations in this study.

\section{Introduction}

The incompressible viscous fluid flow through convergentdivergent channels is one of the most applicable cases in fluid mechanics, civil, environmental, mechanical, and biomechanical engineering. The mathematical investigations of this problem were pioneered by Jeffery [1] and Hamel [2]. They presented an exact similarity solution of the NavierStokes equations in the special case of two-dimensional flow through a channel with inclined plane walls meeting at a vertex and with a source or sink at the vertex and have been extensively studied by several authors and discussed in many textbooks, for example, $[3,4]$. In the Ph.D. thesis [5] we find that Jeffery-Hamel flow used as asymptotic boundary conditions to examine a steady of two-dimensional flow of a viscous fluid in a channel. But, here certain symmetric solutions of the flow has been considered by Sobey and Drazin [6]. Although asymmetric solutions are both possible and of physical interest.

The classical Jeffery-Hamel problem was extended by Axford [7] to include the effects of an external magnetic field on an electrically conducting fluid; in MHD Jeffery-Hamel problems there are two additional nondimensional parameters that determine the solutions, namely, the magnetic
Reynolds number and the Hartmann number. Most scientific problems such as Jeffery-Hamel flows and other fluid mechanic problems are inherently in form of nonlinear differential equations. Except a limited number of these problems, most of them do not have exact solution and some of the solved by numerical methods. Therefore, these nonlinear equations should be solved using other methods. Therefore, many different methods have recently introduced some ways to obtain analytical solution for these nonlinear problems, such as the homotopy perturbation method (HPM) by He $[8,9]$, the homotopy analysis method (HAM) by Liao $[10$, 11], the adomian decomposition method (ADM) [12-14], the variational iteration method (VIM) by He [15], the differential transformation method by Zhou [16], and recently spectral homotopy analysis method (SHAM) by Motsa et al. [17]. In the numerical method, stability and convergence should be considered so as to avoid divergence or inappropriate results. Some of these methods used small parameter in the equation. Therefore, finding the small parameter and exerting it into the equation are deficiencies of these methods.

Nonnumerical approaches include the classical powerseries method and its variants for systems of nonlinear differential equations with small or large embedded parameters such as the homotopy perturbation method. However, it is 
well-known that most of these perturbation solutions are not valid in the whole physical region. These methods do not guarantee the convergence of the series solution, and the perturbation approximations may be only valid for weakly nonlinear problems. Further disadvantages of perturbation methods are that (i) they require the presence of a large or small parameter in the problem while nonperturbation methods require a careful selection of initial approximations and linear operators and (ii) linearization usually leads to difficulties in the integration of higher order deformation equations.

In this work, we present an alternative and improved form of the HPM called spectral-homotopy perturbation method (SHPM) that blends the traditional homotopy perturbation method with the Chebyshev spectral collocation method. The advantage of this approach is that it is more flexible than HPM for choosing a linear operator and initial guess. In HPM, one is restricted to choosing a linear operator and initial approximation that would make the integration of the higher-order differential equations possible whereas the SHPM allows us to have a wider range of selecting linear operators and aninitial guess may be used as long as it satisfies the boundary conditions.

The aim of this study is to apply spectral homotopy perturbation method (SHPM) to find an approximate solution to the nonlinear differential equation governing MHD JefferyHamel flow. We have made a comparison between the current results and other methods with the numerical solution. The results proves the applicability, accuracy, and efficiency of the (SHPM).

\section{Mathematical Formulation}

Consider the steady two-dimensional flow of an incompressible conducting viscous fluid from a source or sink at the intersection between two rigid plane walls that the angel between them is $2 \alpha$. The grid walls are considered to be divergent if $\alpha>0$ and convergent if $\alpha<0$. We assume that the velocity is only along radial direction and depend on $r$ and $\theta$, where $r$ and $\theta$ are radial and angular coordinates, respectively, so that $\mathbf{v}=(u(r, \theta), 0)$ only as shown in Figure 1. Using continuity equation and Navier-Stokes equations in polar coordinates, one has

$$
\begin{gathered}
\quad \frac{\rho}{r} \frac{\partial}{\partial r}(r u(r, \theta))=0 \\
u(r, \theta) \frac{\partial u(r, \theta)}{\partial r} \\
=-\frac{1}{\rho} \frac{\partial P}{\partial r}+v \\
\times\left[\frac{\partial^{2} u(r, \theta)}{\partial r^{2}}+\frac{1}{r} \frac{\partial u(r, \theta)}{\partial r}+\frac{1}{r^{2}} \frac{\partial^{2} u(r, \theta)}{\partial \theta^{2}}-\frac{u(r, \theta)}{r^{2}}\right] \\
-\frac{\sigma B_{0}^{2}}{\rho r^{2}} u(r, \theta), \\
-\frac{1}{\rho r} \frac{\partial P}{\partial \theta}+\frac{2 \nu}{r^{2}} \frac{\partial u(r, \theta)}{\partial \theta}=0
\end{gathered}
$$

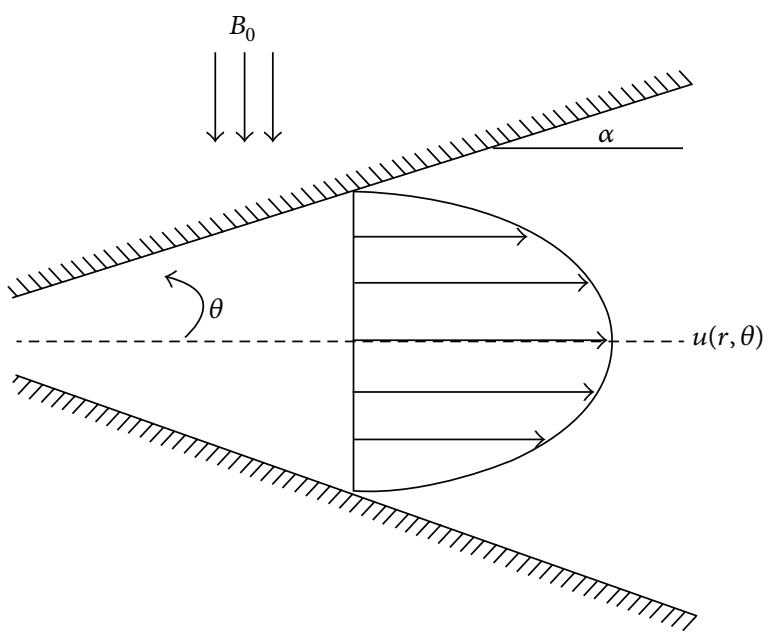

Figure 1: Geometry of the MHD Jeffery-Hamel flow.

where $P$ is the fluid pressure, $B_{0}$ is the electromagnetic induction, $\sigma$ is the conductivity of the fluid, $\rho$ is the fluid density, and $v$ is the coefficient of kinematic viscosity. From (1), one has

$$
u(r, \theta)=\frac{f(\theta)}{r}
$$

Using the dimensionless parameters

$$
F(\eta)=\frac{f(\theta)}{f_{\max }}, \quad \eta=\frac{\theta}{\alpha},
$$

and with eliminating $P$ from (2) and (3), we obtain the following ordinary differential equation for the normalized function profile $F(\eta)$ :

$$
F^{\prime \prime \prime}(\eta)+2 \alpha \operatorname{Re} F(\eta) F^{\prime}(\eta)+(4-H) \alpha^{2} F^{\prime}(\eta)=0,
$$

subject to the boundary conditions

$$
F(0)=1, \quad F^{\prime}(0)=0, \quad F(1)=0,
$$

where Re is the Reynolds number:

$$
\begin{aligned}
\operatorname{Re} & =\frac{\alpha f_{\max }}{\nu} \\
& =\frac{U_{\max } r \alpha}{\nu}=\left(\begin{array}{cc}
\text { divergent channel }: \alpha>0, & U_{\max }>0 \\
\text { convergent channel }: \alpha<0, & U_{\max }<0
\end{array}\right),
\end{aligned}
$$

where $U_{\max }$ is the velocity at the center of the channel $(r=0)$ and $H^{2}=\sigma B_{0}^{2} / \rho \nu$ is the square of the Hartmann number. Now we solve (6) by using (SHPM).

\section{The Homotopy Perturbation Method}

The homotopy perturbation method is a combination of the classical perturbation technique and homotopy technique. 
To illustrate the basic ideas of the HPM, we consider the following nonlinear differential equation:

$$
A(u)-f(\mathbf{r})=0, \quad \mathbf{r} \in \Omega,
$$

with the boundary conditions

$$
B\left(u, \frac{\partial u}{\partial n}\right)=0, \quad \mathbf{r} \in \Gamma,
$$

where $A$ is a general operator, $B$ is a boundary operator, $f(\mathbf{r})$ is a known analytic function, and $\Gamma$ is the boundary of the domain $\Omega$.

The operator $A$ can, generally speaking, be divided into two parts $L$ and $N$, where $L$ is linear, while $N$ is nonlinear, (9), therefore can be written as follows:

$$
L(u)+N(u)-f(\mathbf{r})=0 .
$$

By the homotopy technique (see $[10,11]$ ), we construct a homotopy $v(r, p): \Omega \times[0,1] \rightarrow \mathfrak{R}$ wich satisfies

$$
\begin{aligned}
H(v, p)= & (1-p)\left[L(v)-L\left(u_{0}\right)\right] \\
& +p[A(v)-f(\mathbf{r})]=0, \quad p \in[0,1], \quad \mathbf{r} \in \Omega,
\end{aligned}
$$

or

$$
H(v, p)=L(v)-L\left(u_{0}\right)+p L\left(u_{0}\right)+p[N(v)-f(\mathbf{r})]=0,
$$

where $p \in[0,1]$ is an embedding parameter and $u_{0}$ is an initial approximation of (9), wich satisfies the boundary conditions. Obviously, from (12) we have

$$
\begin{aligned}
& H(v, 0)=L(v)-L\left(u_{0}\right)=0, \\
& H(v, 1)=A(v)-f(\mathbf{r})=0 .
\end{aligned}
$$

The changing process of $p$ from 0 to 1 is just that of $v(\mathbf{r}, p)$ from $u_{0}(\mathbf{r})$ to $u(\mathbf{r})$. In topology, this is called deformation, and $L(v)-L\left(u_{0}\right), A(v)-f(\mathbf{r})$ are called homotopic.

According to HPM, we can first use the embedding parameter $p$ as small parameter and assume that the solution of (12) can be written as a power series ip $p 4$ :

$$
v=v_{0}+p v_{1}+p^{2} v_{2}+\cdots
$$

setting $p=1$, results in the approximation to the solution of (9)

$$
u=\lim _{p \rightarrow 1} v=v_{0}+v_{1}+v_{2}+\cdots
$$

The series (16) is convergent for most cases; however, the convergent depends upon the nonlinear operator $A(v)$. Some criteria suggested for convergence of the series (16) and the following opinions are suggested by $\mathrm{He}[18,19]$.

(1) The second derivative of $N(v)$ with respect to $v$ must be small because the parameter $p$ may be relatively large, that is, $p \rightarrow 1$.

(2) The norm of $L_{-1}(\partial N / \partial v)$ must be smaller than one so that the series converges.

\section{The Spectral Homotopy Perturbation Method}

To solve the nonlinear ordinary differential equation (6) using the SHPM we start by transfom the domain of the problem from $[0,1]$ to the domain $[-1,1]$ on which the Chebyshev spectral method can be implemented, using the algebraic mapping

$$
x=2 \eta-1, \quad x \in[-1,1] .
$$

It is also convenient to make the boundary conditions homogeneous by making use of the transformation

$$
F(\eta)=f(x)+1-\eta^{2} .
$$

Substituting (18) and (17) in (6) and the boundary conditions (7) gives

$$
\begin{aligned}
f^{\prime \prime \prime}(x) & +g_{1}(x) f^{\prime}(x)+g_{2}(x) f(x) \\
& +\frac{1}{2} \alpha \operatorname{Re} f(x) f^{\prime}(x)=G(x),
\end{aligned}
$$

subject to

$$
f(-1)=f(1)=f^{\prime}(-1)=0,
$$

where

$$
\begin{aligned}
g_{1}(x)= & \frac{1}{8}\left(3-2 x-x^{3}\right)+\frac{1}{4} \alpha^{2}(2+2 x-H), \\
g_{2}(x)= & -\frac{1}{4} \alpha \operatorname{Re}(x+1), \\
G(x)= & \frac{1}{16} \alpha \operatorname{Re}(x+1)\left(3-2 x-x^{2}\right) \\
& +\frac{1}{8} \alpha^{2}(x+1)(4-H) .
\end{aligned}
$$

To apply the SHPM to the differential equation (19) we may choose the following linear differential operator:

$$
\mathscr{L}=\frac{d^{3}}{d x^{3}}+g_{1}(x) \frac{d}{d x}+g_{2}(x) .
$$

The initial approximation for the solution of (19) is obtained from the solution to the linear part of (19). Consider

$$
f_{0}^{\prime \prime \prime}(x)+g_{1}(x) f_{0}^{\prime}+g_{2}(x) f_{0}(x)=G(x),
$$

subject to the boundary conditions

$$
f_{0}(-1)=f_{0}(1)=f_{0}^{\prime}(-1)=0 .
$$

If an exact solution of (23) cannot be found, we use the Chebyshev pseudospectral method to solve the equation. We now construct the homotopy:

$$
\begin{aligned}
H(F ; p)= & \mathscr{L}[F]-\mathscr{L}\left[f_{0}\right]+p \mathscr{L}\left[f_{0}\right] \\
& +p(N(F)-G(x))=0
\end{aligned}
$$


where $F$ is an approximate series solution of given by

$$
F=f_{0}+p f_{1}+p^{2} f_{2}+\cdots .
$$

And $N(F)$ is the nonlinear part of (19). Substitute (22) in (25) to get

$$
\begin{aligned}
\frac{d^{3} F}{d x^{3}} & +g_{1}(x) \frac{d F}{d x}+g_{2}(x) F+(1-p) \\
& \times\left(\frac{d^{3} f_{0}}{d x^{3}}+g_{1}(x) \frac{d f_{0}}{d x}+g_{2}(x) f_{0}\right) \\
& +p\left(\frac{1}{2} \alpha \operatorname{Re} F \frac{d F}{d x}-G(x)\right) \\
= & 0 .
\end{aligned}
$$

We make a comparison between the power of $p^{1}$ in both sides of (27) to obtain the following system of equations:

$$
\mathbf{A} f_{1}=\mathbf{B}_{1},
$$

subject to the boundary conditions

$$
f_{1}(-1)=f_{1}(1)=f_{1}(-1)=0,
$$

where

$$
\begin{aligned}
& \mathbf{A}=\left[\mathscr{D}^{3}+\operatorname{diag}\left[g_{1}\left(x_{i}\right)\right] \mathscr{D}+\operatorname{diag}\left[g_{2}\left(x_{i}\right)\right]\right], \\
& \mathbf{B}_{\mathbf{1}}=-\left[\mathbf{A} f_{0}+\frac{1}{2} \alpha \operatorname{Re} f_{0}\left(\mathscr{D}^{2} f_{0}\right)-G\left(x_{i}\right)\right]^{T},
\end{aligned}
$$

where $T$ denotes transpose, diag [] is a diagonal matrix of size $(N+1) \times(N+1)$, and $\mathscr{D}$ is the Chebyshev spectral differentiation matrix whose entries (see $[20,21])$ are given by

$$
\begin{aligned}
& \mathscr{D}_{j k}=\frac{c_{j}}{c_{k}} \frac{(-1)^{j+k}}{x_{j}-x_{k}} \quad j \neq k ; j, k=0,1, \ldots, N, \\
& \mathscr{D}_{k k}=-\frac{x_{k}}{2\left(1-x_{k}^{2}\right)} \quad k=1,2, \ldots, N-1, \\
& \mathscr{D}_{00}=\frac{2 N^{2}+1}{6}=-\mathscr{D}_{N N} .
\end{aligned}
$$

Here $c_{0}=c_{N}=2$ and $c_{j}=1$ with $1 \leq j \leq N-1, x_{j}$ are the Chebyshev collocation points (see [20]) defined by

$$
x_{j}=\cos \frac{j \pi}{N}, \quad j=0,1,2 \ldots, N,
$$

the solution of (28) can be given by

$$
f_{1}=\mathbf{A}^{-1} \mathbf{B}_{\mathbf{1}} .
$$

To get more higher order approximations for (19), we compare between the coefficients of $p^{i},(i=2,3,4, \ldots)$ in (25) to obtain the following approximations:

$$
f_{i}=\mathbf{A}^{-1} \mathbf{B}_{\mathbf{i}}
$$

subject to the boundary conditions

$$
f_{i}(-1)=f_{i}(1)=f_{i}^{\prime}(-1)=0,
$$

where

$$
\mathbf{B}_{\mathbf{i}}=-\frac{1}{2} \alpha \operatorname{Re}\left[\sum_{n=0}^{i-1} f_{n}\left(\mathscr{D} f_{i-1-n}\right)\right], \quad i \geq 2 .
$$

The matrix $A$ has dimensions $(N+1) \times(N+1)$ while matrices $B_{i}$ and $f_{i}$ have a dimensions $(N+1) \times 1$.

To implement the boundary conditions (29) and (35) to the systems (28) and (34), respectively, we delete the first and the last rows and columns of $A$ and delete the first and last rows of and $f_{1}$ and $f_{i}$; also we replace the results of last row of the modified matrix $A$ and setting the results of last row of the modified matrices $B_{1}$ and $B_{i}$ to be zero. Then the solution of (19) is given by substituting the series $f_{i}$ in (26) after setting $p=1$.

\section{Results and Discussion}

In this section we present the obtained results of the solutions for MHD Jeffery-Hame flow using the HPM, SHPM, and a numerical solution. Here we used the inbuilt MATLAB boundary value problems solver bvp4c for the numerical solution approach. In generating the presented results it was determined through numerical experimentation that $N=$ 60. Table 1 shows a comparison between the HPM, SHPM, and numerical approximate solutions of $F(\eta)$. The table shows that the results of the present method are in excellent agreement with those of the numerical ones. Also in Table 2 we give a comparison of the SHPM results for divergent and convergent channels and fixed values of $H$ and Re when $\eta$ is varied at different orders of approximation against the numerical results. It can be seen from Table 2 that SHPM results converge rapidly to the numerical solution. Table 3 gives a comparison of the differential transformation method (DTM), HPM, homotopy analysis method (HAM) given by Joneidi et al. [22], and SHPM results for $F(\eta)$ against the numerical results when $\eta$ is varied. It can be seen from this table that the approximate solution of MHD Jeffery-Hamel flows obtained by SHPM is very accurate and it is converges much more rapidly to the numerical result compared to the DTM, HPM, and HAM.

Figures 2(a) and 2(b) show firstly the influence of the magnetic field parameter on the velocity profile for divergent and convergent channels and, secondly, a comparison between the present results and numerical results to give a sense of the accuracy and convergence rate of the SHPM. The figures indicate that there is very good match between the two sets of results even at very low orders of SHPM approximations series compared with the numerical results. These findings firmly establish the SHPM as an accurate and alternative to the HPM. Also it can seen that the fluid velocity increases with increasing Hartman numbers for both convergent and divergent channels. 
TABLE 1: Comparison between the HPM, SHPM, and numerical results of $F(\eta)$ for different values of $H$ when $\alpha=7.5^{\circ}$ and Re $=50$.

\begin{tabular}{|c|c|c|c|c|c|c|c|c|c|c|}
\hline & $\eta$ & 0.1 & 0.2 & 0.3 & 0.4 & 0.5 & 0.6 & 0.7 & 0.8 & 0.9 \\
\hline \multirow{3}{*}{$H=0$} & HPM & 0.977071 & 0.911454 & 0.810411 & 0.685923 & 0.549843 & 0.413170 & 0.284602 & 0.170279 & 0.074423 \\
\hline & SHPM & 0.977136 & 0.911454 & 0.810955 & 0.686840 & 0.551195 & 0.415007 & 0.286958 & 0.173044 & 0.076826 \\
\hline & Numeric & 0.977136 & 0.911453 & 0.810954 & 0.686838 & 0.551193 & 0.415005 & 0.286957 & 0.173044 & 0.076826 \\
\hline \multirow{3}{*}{$H=250$} & HPM & 0.983734 & 0.936343 & 0.861689 & 0.765340 & 0.653396 & 0.531462 & 0.403813 & 0.272871 & 0.138943 \\
\hline & SHPM & 0.983736 & 0.936343 & 0.861708 & 0.765375 & 0.653451 & 0.531542 & 0.403921 & 0.273000 & 0.139045 \\
\hline & Numeric & 0.983736 & 0.936343 & 0.861708 & 0.765375 & 0.653451 & 0.531542 & 0.403921 & 0.273000 & 0.139045 \\
\hline \multirow{3}{*}{$H=500$} & HPM & 0.988320 & 0.953804 & 0.897852 & 0.822470 & 0.729682 & 0.620975 & 0.496664 & 0.355212 & 0.192382 \\
\hline & SHPM & 0.988322 & 0.953804 & 0.897870 & 0.822501 & 0.729725 & 0.621028 & 0.496723 & 0.355266 & 0.192418 \\
\hline & Numeric & 0.988322 & 0.953804 & 0.897870 & 0.822501 & 0.729725 & 0.621028 & 0.496723 & 0.355266 & 0.192418 \\
\hline \multirow{3}{*}{$H=1000$} & HPM & 0.993761 & 0.974806 & 0.942279 & 0.894743 & 0.829747 & 0.743476 & 0.629987 & 0.479934 & 0.278279 \\
\hline & SHPM & 0.993765 & 0.974806 & 0.942318 & 0.894809 & 0.829843 & 0.743602 & 0.630138 & 0.480096 & 0.278418 \\
\hline & Numeric & 0.993765 & 0.974806 & 0.942318 & 0.894809 & 0.829843 & 0.743602 & 0.630138 & 0.480096 & 0.278418 \\
\hline
\end{tabular}

TABLE 2: Comparison of the values of the SHPM approximate solutions for $F(\eta)$ with the numerical solution for various values of $\eta$ when $H=100$ and $\operatorname{Re}=50$ for divergent and convergent channels.

\begin{tabular}{|c|c|c|c|c|c|c|c|c|c|c|}
\hline \multirow{2}{*}{$\eta$} & \multicolumn{5}{|c|}{ divergent-channel $\alpha=5^{\circ}$} & \multicolumn{5}{|c|}{ convergent-channel $\alpha=-5^{\circ}$} \\
\hline & 3rd order & 4 th order & 6 th order & 8th order & Numerical & 3rd order & 4 th order & 6 th order & 8th order & Numerical \\
\hline 0.1 & 0.983499 & 0.983500 & 0.983500 & 0.983500 & 0.983500 & 0.994672 & 0.994672 & 0.994672 & 0.994672 & 0.994672 \\
\hline 0.2 & 0.935285 & 0.935287 & 0.935286 & 0.935286 & 0.935286 & 0.978184 & 0.978184 & 0.978184 & 0.978184 & 0.978184 \\
\hline 0.3 & 0.858989 & 0.858991 & 0.858991 & 0.858991 & 0.858991 & 0.948987 & 0.948986 & 0.948986 & 0.948986 & 0.948986 \\
\hline 0.4 & 0.759953 & 0.759957 & 0.759957 & 0.759957 & 0.759957 & 0.904400 & 0.904399 & 0.904399 & 0.904399 & 0.904399 \\
\hline 0.5 & 0.644378 & 0.644384 & 0.644384 & 0.644384 & 0.644384 & 0.840477 & 0.840475 & 0.840475 & 0.840475 & 0.840475 \\
\hline 0.6 & 0.518442 & 0.518448 & 0.518448 & 0.518448 & 0.518448 & 0.751876 & 0.751874 & 0.751874 & 0.751874 & 0.751874 \\
\hline 0.7 & 0.387576 & 0.387581 & 0.387581 & 0.387581 & 0.387581 & 0.631800 & 0.631798 & 0.631798 & 0.631798 & 0.631798 \\
\hline 0.8 & 0.255978 & 0.255981 & 0.255981 & 0.255981 & 0.255981 & 0.472168 & 0.472166 & 0.472166 & 0.472166 & 0.472166 \\
\hline 0.9 & 0.126379 & 0.126380 & 0.126380 & 0.126380 & 0.126380 & 0.264232 & 0.264231 & 0.264231 & 0.264231 & 0.264231 \\
\hline
\end{tabular}

TABLE 3: Comparison between DTM, HPM, HAM, SHPM, and numerical solution of $F(\eta)$ for various values of $\eta$ when $\operatorname{Re}=80, \alpha=-5^{\circ}$, and $H=0$.

\begin{tabular}{lccccc}
\hline$\eta$ & ADM & HPM & HAM & SHPM & Numerical \\
\hline 0.0 & 1 & 1 & 1 & 1 & 0.99596206 \\
0.1 & 0.99596039 & 0.99606719 & 0.99596062 & 0.98328122 & 0.99596205 \\
0.2 & 0.98327455 & 0.98369594 & 0.98327553 & 0.96019253 & 0.98328121 \\
0.3 & 0.96017756 & 0.96107588 & 0.96017989 & 0.92354347 & 0.96019253 \\
0.4 & 0.92351707 & 0.92492452 & 0.92352157 & 0.86849152 & 0.92354347 \\
0.5 & 0.86845113 & 0.87019977 & 0.86845890 & 0.78813414 & 0.78813414 \\
0.6 & 0.78807854 & 0.78983259 & 0.78809102 & 0.67319429 & 0.67319429 \\
0.7 & 0.67312484 & 0.67453350 & 0.67314377 & 0.51204134 & 0.51204134 \\
0.8 & 0.51196441 & 0.51283731 & 0.51199099 & 0.29159436 & 0.29159436 \\
0.9 & 0.29152801 & 0.29189370 & 0.29155802 & 0.00000000 & 0.00000000 \\
1.0 & 0.00000000 & 0.00000000 & -0.00000115 & &
\end{tabular}

\section{Conclusions}

In this study, the SHPM were applied successfully to find an approximate solution of a nonlinear MHD Jeffery-Hamel flows. The effect of the magnetic field parameter on the velocity profile for convergent and divergent channels has been determined. It could be noticed that by increasing magnetic field parameter, the velocity profile increases resulting in a rise in the flow rate for both convergent and divergent channels. The obtained results compared with the numerical solution of the governing nonlinear equation and with DTM, HPM, and HAM. Also the tables and figures clearly show high accuracy of the method and the convergent is very fast to solve MHD Jeffery-Hamel problem. An important aspect of this work has been the need to prove the computational efficiency and accuracy of the SHPM in solving nonlinear 


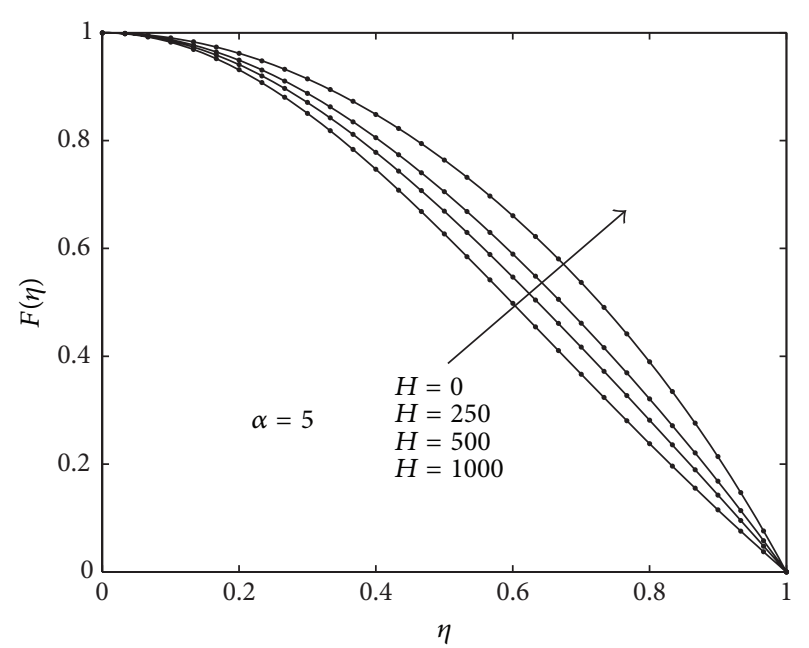

(a)

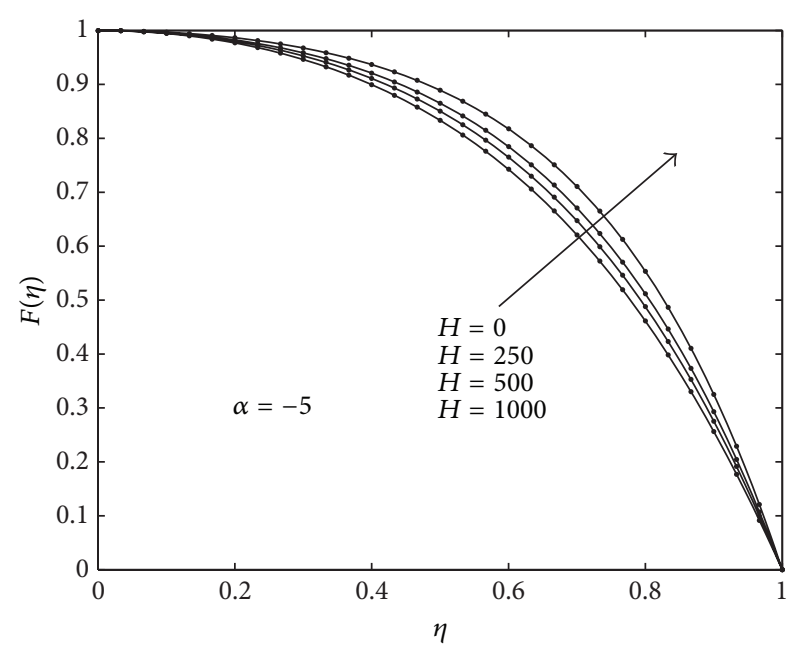

(b)

FIGURE 2: Comparison between the numerical solution and SHPM of $F(\eta)$ for divergent and convergent channels when Re $=50$.

differential equations and this method is, in general, more accurate than the HPM. The results in this paper confirm that the SHPM is a powerful and efficient technique for finding solutions for nonlinear differential equations in different fields of science and engineering.

\section{Conflict of Interests}

The author declares that there is no conflict of interests regarding the publication of this paper.

\section{References}

[1] G. B. Jeffery, "The two-dimensional steady motion of a viscous fluid," Philosophical Magazine, vol. 6, pp. 455-465, 1915.

[2] G. Hamel, "Spiralförmige Bewegungen zäher Flüssigkeiten," Jahresbericht der Deutschen Mathematiker-Vereinigung, vol. 25, pp. 34-60, 1916.

[3] L. Rosenhead, "The steady two-dimensional radial flow of viscous fluid between two inclined plane walls," Proceedings of the Royal Society A, vol. 175, pp. 436-467, 1940.

[4] A. McAlpine and P. G. Drazin, "On the spatio-temporal development of small perturbations of Jeffery-Hamel flows," Fluid Dynamics Research, vol. 22, no. 3, pp. 123-138, 1998.

[5] R. M. Sadri, Channel entrance flow [Ph.D. thesis], Department of Mechanical Engineering, the University of Western Ontario, 1997.

[6] I. J. Sobey and P. G. Drazin, "Bifurcations of two-dimensional channel flows," Journal of Fluid Mechanics, vol. 171, pp. 263-287, 1986.

[7] W. I. Axford, "The magnetohydrodynamic Jeffrey-Hamel problem for a weakly conducting fluid," The Quarterly Journal of Mechanics and Applied Mathematics, vol. 14, pp. 335-351, 1961.

[8] J. He, "A review on some new recently developed nonlinear analytical techniques," International Journal of Nonlinear Sciences and Numerical Simulation, vol. 1, no. 1, pp. 51-70, 2000.
[9] J. H. He, "Homotopy perturbation method for bifurcation of nonlinear problems," International Journal of Nonlinear Sciences and Numerical Simulation, vol. 8, no. 2, pp. 207-208, 2005.

[10] S. J. Liao, The proposed homotopy analysis technique for the solution of nonlinear problems [Ph.D. thesis], Shanghai Jiao Tong University, 1992.

[11] S. Liao, "On the homotopy analysis method for nonlinear problems," Applied Mathematics and Computation, vol. 147, no. 2, pp. 499-513, 2004.

[12] Q. Esmaili, A. Ramiar, E. Alizadeh, and D. D. Ganji, "An approximation of the analytical solution of the Jeffery-Hamel flow by decomposition method," Physics Letters A: General, Atomic and Solid State Physics, vol. 372, no. 19, pp. 3434-3439, 2008.

[13] O. D. Makinde and P. Y. Mhone, "Hermite-Padé approximation approach to MHD Jeffery-Hamel flows," Applied Mathematics and Computation, vol. 181, no. 2, pp. 966-972, 2006.

[14] O. D. Makinde, "Effect of arbitrary magnetic Reynolds number on MHD flows in convergent-divergent channels," International Journal of Numerical Methods for Heat \& Fluid Flow, vol. 18, no. 5-6, pp. 697-707, 2008.

[15] J. H. He, "Variational iteration method-a kind of non-linear analytical technique: Some examples," International Journal of Non-Linear Mechanics, vol. 34, no. 4, pp. 699-708, 1999.

[16] J. K. Zhou, Differential Transformation and Its Applications for Electrical Circuits, Huazhong University Press, Wuhan, China, 1986, (Chinese).

[17] S. S. Motsa, P. Sibanda, F. G. Awad, and S. Shateyi, "A new spectral-homotopy analysis method for the MHD JefferyHamel problem," Computers \& Fluids, vol. 39, no. 7, pp. 1219$1225,2010$.

[18] J. H. He, "Homotopy perturbation technique," Computer Methods in Applied Mechanics and Engineering, vol. 178, no. 3-4, pp. 257-262, 1999.

[19] J. H. He, "A coupling method of a homotopy technique and a perturbation technique for non-linear problems," International Journal of Non-Linear Mechanics, vol. 35, no. 1, pp. 37-43, 2000.

[20] L. N. Trefethen, Spectral Methods in MATLAB, SIAM, 2000. 
[21] W. S. Don and A. Solomonoff, "Accuracy and speed in computing the Chebyshev collocation derivative," SIAM Journal on Scientific Computing, vol. 16, no. 6, pp. 1253-1268, 1995.

[22] A. A. Joneidi, G. Domairry, and M. Babaelahi, "Three analytical methods applied to Jeffery-Hamel flow," Communications in Nonlinear Science and Numerical Simulation, vol. 15, no. 11, pp. 3423-3434, 2010. 

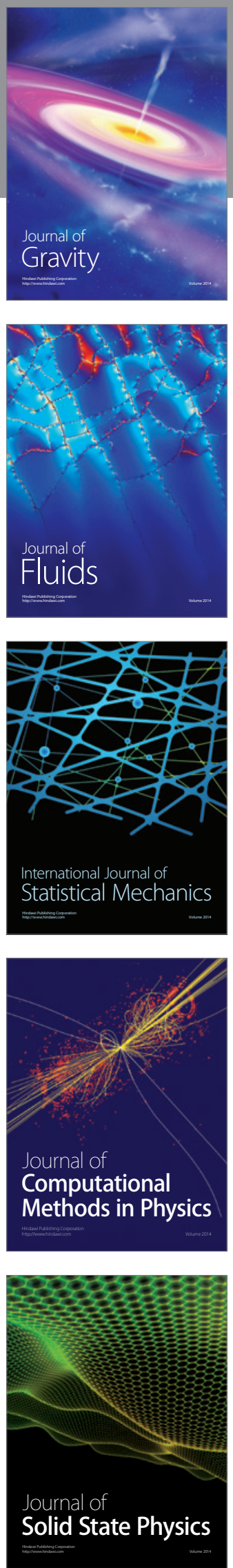

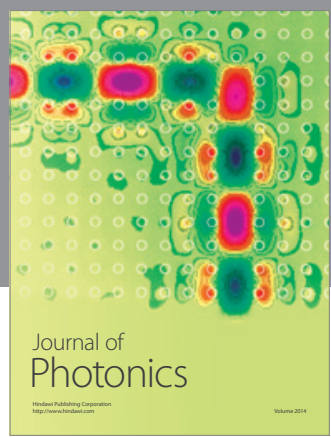

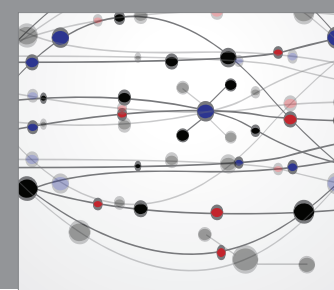

The Scientific World Journal

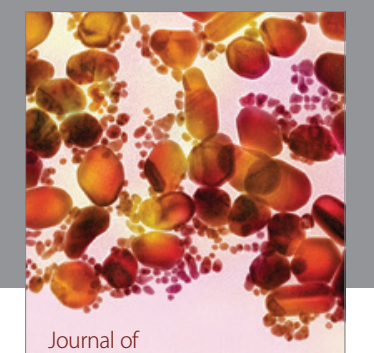

Soft Matter
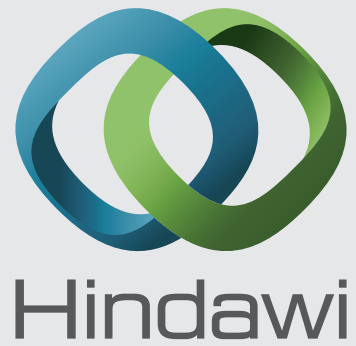

Submit your manuscripts at

http://www.hindawi.com
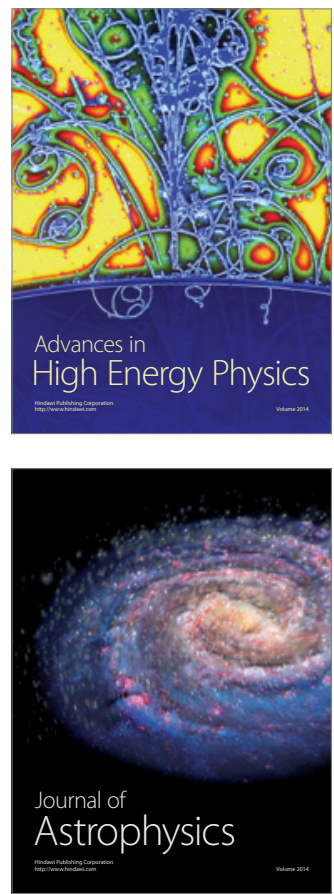
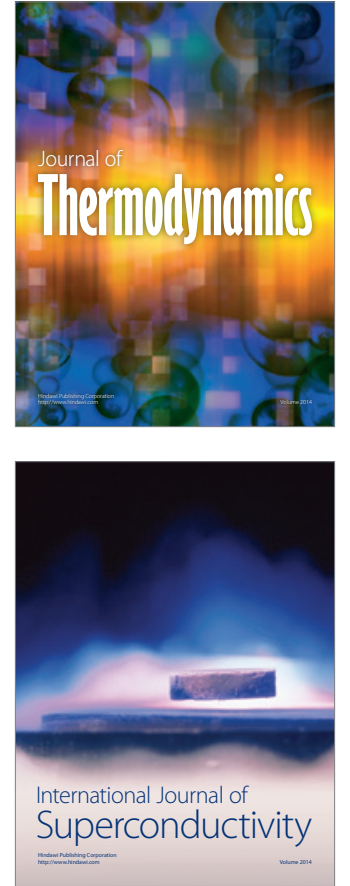
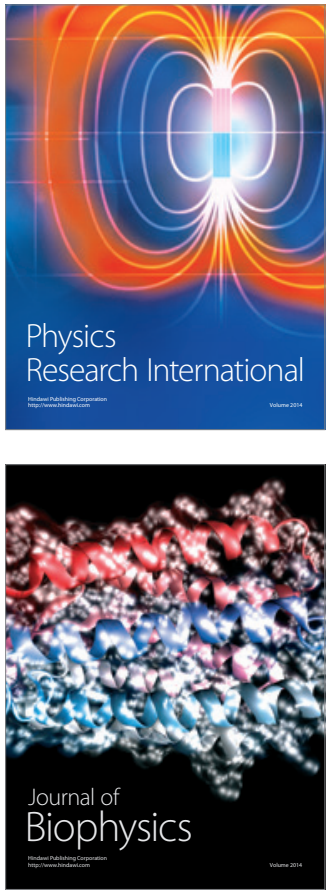
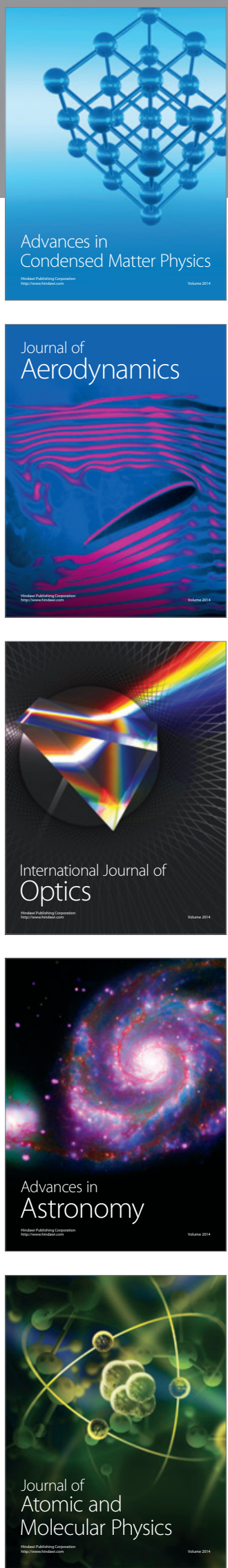